\title{
A Final Word on FCNC-Baryogenesis from Two Higgs Doublets
}

\author{
Wei-Shu Hou ${ }^{1}$, Tanmoy Modak ${ }^{2}$, and Tilman Plehn² \\ 1 Department of Physics, National Taiwan University, Taipei, Taiwan \\ 2 Institut für Theoretische Physik, Universität Heidelberg, Germany \\ modak@thphys.uni-heidelberg.de
}

February 25, 2021

\begin{abstract}
Electroweak baryogenesis in a two-Higgs doublet model is a well-motivated and testable scenario for physics beyond the Standard Model. One attractive way of providing $C P$ violation is through flavor-changing Higgs couplings, where a link between top and charm quarks is hardly affected by flavor and $C P$-violation constraints. This scenario can be tested by searching for heavy charged and neutral Higgs bosons at the LHC. While the charged Higgs signature requires a dedicated analysis, the neutral Higgs signature will be covered by a general search for same-sign top pairs. Together, they provide a conclusive test of the flavor-changing couplings required for this kind of baryogenesis.
\end{abstract}

Content

\begin{tabular}{lll}
\hline 1 & Introduction & 2
\end{tabular}

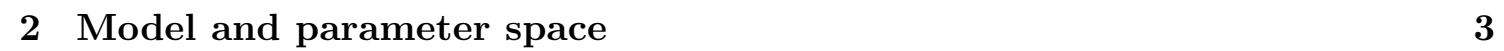

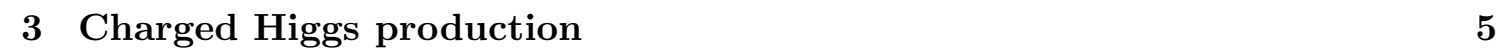

\begin{tabular}{|llr}
\hline 4 & Neutral Higgs production & 8 \\
\hline
\end{tabular}

\begin{tabular}{lll}
\hline 5 Outlook & 11
\end{tabular}

\begin{tabular}{ll}
\hline References & 12
\end{tabular} 


\section{Introduction}

The Higgs discovery [1,2] and subsequent measurements of the Higgs Lagrangian [3 11] indicate that the Standard Model is the correct effective theory around the electroweak scale. While there exists no experimental evidence for physics beyond the Standard Model so far, extended Higgs sectors are motivated by theoretical considerations, like mass generation of up-type and down-type fermions, neutrino mass generation, electroweak baryogenesis, or dark matter. In particular, two-Higgs doublet models (2HDMs) 12 14] are an integral part of well-defined models for physics beyond the Standard Model, including MSSM [15], composite Higgs models [16, little Higgs models [17, 18, or GUTs 19 22.

From an electroweak baryogenesis point of view [23], a 2HDM can provide both new scalar degrees of freedom 24, 25] and $C P$-violation. In the general [26, 27] or type-III [28] model, the additional states can be close in mass to the SM-Higgs [29, 30]. Starting with two doublets, both coupling to up-type and down-type quarks hence two separate Yukawa matrices, we diagonalize the quark mass matrices and find the real, diagonal coupling $\lambda_{i i}=$ $\sqrt{2} m_{i} / v$ and the complex, non-diagonal coupling $\rho_{i j}$. Flavor-changing neutral couplings are generally constrained, but it is possible to have electroweak baryogenesis (EWBG) driven by an order-one, complex coupling $\rho_{t c}$. A valid parameter region is 31]

$$
\operatorname{Im} \rho_{t c} \gtrsim 0.5 \quad \text { and } \quad|\cos \gamma| \gtrsim 0.1
$$

where $\gamma$ is the mixing angle between the two $C P$-even Higgs states.

It has been shown 32,33 (see also Refs. 34,36$]$ ) that the coupling $\rho_{t c}$ can be discovered in the LHC process

$$
c g \rightarrow t A / H \rightarrow t(t \bar{c})
$$

where this process retains a mild dependence on $\cos \gamma$ and is especially useful for small values of $\cos \gamma$. To attribute this signal to EWBG requires information on the mixing angle $\cos \gamma$. A promising LHC search channel to test this kind of scenario is $b$-associated charged Higgs production 37

$$
c g \rightarrow b H^{+} \rightarrow b\left(W^{+} h\right) .
$$

Whereas the production process is induced by $\rho_{t c}$ 38, 39, the decay amplitude is proportional to the mixing angle $\cos \gamma$. Even in the absence of complex phase information, such a search can test precisely the underlying $\rho_{t c}$-EWBG scenario. Finally, the exotic top decay

$$
t \rightarrow c h
$$

is induced by the coupling $\rho_{t c}$ combined with non-vanishing $\cos \gamma[28$. It is searched for by CMS [40] and ATLAS [41].

In this paper we show how the two LHC searches for charged and neutral heavy Higgs bosons can conclusively probe the parameter region required for $\rho_{t c}$-EWBG in the general 2HDM (g2HDM). The paper is organized as follows: in Sec. 2 we discuss the model and its preferred parameter space, and then compare it to the reach of the charged Higgs channel in Sec. 3. Section 4 is dedicated to same-sign top production from neutral Higgs production and its complementarity to the charged Higgs signature. We summarize our results in Sec. 5 , 


\section{Model and parameter space}

The general $C P$-conserving two Higgs doublet potential can be written as [42, 43]

$$
\begin{aligned}
V\left(\Phi, \Phi^{\prime}\right)= & \mu_{11}^{2}|\Phi|^{2}+\mu_{22}^{2}\left|\Phi^{\prime}\right|^{2}-\left(\mu_{12}^{2} \Phi^{\dagger} \Phi^{\prime}+\text { h.c. }\right)+\frac{\eta_{1}}{2}|\Phi|^{4}+\frac{\eta_{2}}{2}\left|\Phi^{\prime}\right|^{4}+\eta_{3}|\Phi|^{2}\left|\Phi^{\prime}\right|^{2} \\
& +\eta_{4}\left|\Phi^{\dagger} \Phi^{\prime}\right|^{2}+\left[\frac{\eta_{5}}{2}\left(\Phi^{\dagger} \Phi^{\prime}\right)^{2}+\left(\eta_{6}|\Phi|^{2}+\eta_{7}\left|\Phi^{\prime}\right|^{2}\right) \Phi^{\dagger} \Phi^{\prime}+\text { h.c. }\right] .
\end{aligned}
$$

In the Higgs basis, the VEV $v=246 \mathrm{GeV}$ is generated by the doublet $\Phi$, while $\Phi^{\prime}$ does not develop a VEV, hence $\mu_{22}^{2}>0$. The minimization conditions in the two field directions lead to $\mu_{11}^{2}=-\eta_{1} v^{2} / 2$ and $\mu_{12}^{2}=\eta_{6} v^{2} / 2$. The mixing angle $\gamma$ diagonalizes the $C P$-even mass matrix to define the mass eigenstates $h$ and $H$ [42, 43], satisfying the relations

$$
c_{\gamma}^{2}=\cos ^{2} \gamma=\frac{\eta_{1} v^{2}-m_{h}^{2}}{m_{H}^{2}-m_{h}^{2}} \quad \text { and } \quad s_{2 \gamma}=\sin (2 \gamma)=\frac{2 \eta_{6} v^{2}}{m_{H}^{2}-m_{h}^{2}}
$$

simultaneously, where $c_{\gamma} \rightarrow 0$ in the alignment limit $\left(\eta_{6}=0\right.$ and $\left.\eta_{1}=m_{h}^{2} / v^{2} \sim 1 / 4\right)$. As one of the ingredients to baryogenesis, a strong first-order phase transition can be triggered by a second scalar degree of freedom close in mass with the SM-Higgs 4446 . From Eq. (6) we see that this is guaranteed by finite $c_{\gamma}$ and perturbatively stable $\eta_{i}=\mathcal{O}(1)$, for instance $\eta_{6}=\mathcal{O}(1)$ and $\eta_{1}=\mathcal{O}(1)>m_{h}^{2} / v^{2}[43$.

For baryogenesis, we need a complex phase in the Higgs or Yukawa sectors of the general 2HDM. Many analyses have studied a complex Higgs potential, which tends to be strongly constrained by EDM measurements [47 50]. We look at the alternative option of $C P$-violation arising from the Yukawa sector $31,42,51$

$$
\begin{aligned}
\mathcal{L} \supset & -\frac{1}{\sqrt{2}} \sum_{F=U, D, L} \bar{F}_{i}\left[\left(-\lambda_{i j}^{F} s_{\gamma}+\rho_{i j}^{F} c_{\gamma}\right) h+\left(\lambda_{i j}^{F} c_{\gamma}+\rho_{i j}^{F} s_{\gamma}\right) H-i \operatorname{sgn}\left(Q_{F}\right) \rho_{i j}^{F} A\right] P_{R} F_{j} \\
& -\bar{U}_{i}\left[\left(V \rho^{D}\right)_{i j} P_{R}-\left(\rho^{U \dagger} V\right)_{i j} P_{L}\right] D_{j} H^{+}-\bar{\nu}_{i} \rho_{i j}^{L} P_{R} L_{j} H^{+}+\text {h.c. },
\end{aligned}
$$

where $i, j=1,2,3$ are generation indices, $P_{L, R} \equiv\left(1 \mp \gamma_{5}\right) / 2$, and $V$ is the CKM matrix. In flavor space, the fermion fields $F$ are defined as $U=(u, c, t), D=(d, s, b), L=(e, \mu, \tau)$ and $\nu=\left(\nu_{e}, \nu_{\mu}, \nu_{\tau}\right)$. While the mass matrices are diagonalized as in the Standard Model, one cannot rotate away $C P$-violating phases of the second set of $\rho^{F}$ matrices in the general $2 \mathrm{HDM}$ even in the Higgs basis. That is, the two coupling matrices are

$$
\lambda_{i j}^{F}=\sqrt{2} \frac{m_{i}^{F}}{v} \delta_{i j} \in \mathbb{R} \quad \text { and } \quad \rho_{i j}^{F} \in \mathbb{C} .
$$

The complex coupling matrices $\rho^{F}$ are, strictly speaking, not related to the fermion masses. On the other hand, given experimental constraints and a possible order-of-magnitude correspondence in the values of $\rho^{F}$ and $\lambda^{F}$ lead us to consider $\rho_{t j}^{U}$ or $\rho_{t t}^{U}$. As our primary focus is to probe the parameter space required for $\rho_{t c}$-EWBG, we turn off all $\rho_{i j}$ couplings except for $\rho_{t c}$. However, we shall return to the impact of non-vanishing $\rho_{i j}$ later.

In principle, a finite imaginary part of $\rho_{t t}$ can quite robustly drive EWBG 31, which motivates search for channels like $g g \rightarrow H \rightarrow t \bar{t}$ or $g g \rightarrow H t \bar{t} \rightarrow 4 t$ [52 54]. We focus instead on complex off-diagonal entries $\rho_{t j}$, specifically $\rho_{t c}$. With a large phase, this FCNC coupling can also [31] drive EWBG and account for the observed baryon asymmetry if $\rho_{t t}$ turns out to be small. One merit of the $\rho_{t c}$ mechanism for EWBG is that $\rho_{t c}$ does not generate an electron EDM through the Barr-Zee [55] two-loop mechanism, and can 

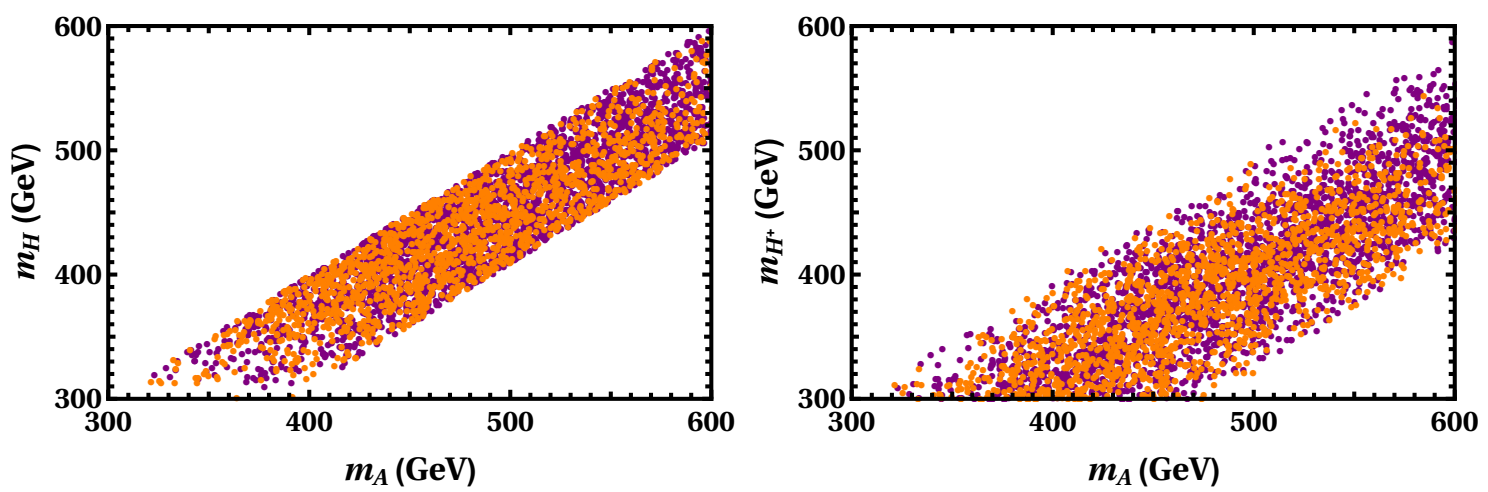

Figure 1: Parameter space allowed by perturbativity, positivity, unitarity, and electroweak precision measurements in the $m_{A}-m_{H}$ and $m_{A^{-}} m_{H^{ \pm}}$planes. The purple and orange scanned points corresponds to $c_{\gamma}=0.1$ and 0.3 respectively.

therefore more easily $[56]$ evade the ACME bound $[57] d_{e}<1.1 \times 10^{-29} e \mathrm{~cm}$. Moreover, as we assumed $\rho_{c t}$ to be vanishingly small, the constraint on $\rho_{t c}$ from charm chromo-EDM is also not stringent (see e.g. discussion in Ref. [58]). In particular, for $\left|\rho_{t t}\right| \lesssim 10^{-2}$ the baryon asymmetry can still be generated if 31

$$
\left|\rho_{t c}\right| \gtrsim 0.5 \quad \text { and } \quad\left|c_{\gamma}\right| \gtrsim 0.1,
$$

assuming a sufficiently large complex phase. We remind ourselves that a strong first-order phase transition is possible for 5968

$$
m_{A, H, H^{+}} \sim 300 \ldots 600 \mathrm{GeV} .
$$

This mass range is allowed by perturbativity, positivity, unitarity, and electroweak precision data. We rely on 2HDMC [69] to provide the results of Fig. 1 for $c_{\gamma}=0.1$ and 0.3. The $2 \mathrm{HDMC}$ parameters in the Higgs basis are $\eta_{1, . ., 7}$ and $m_{H^{ \pm}}$. To save computing time we actually scan $\mu_{22} \in[0,1000] \mathrm{GeV}, m_{A} \in[300,600] \mathrm{GeV}, m_{H} \in[300,600] \mathrm{GeV}$, $m_{H^{ \pm}} \in[300,600] \mathrm{GeV}, \eta_{2} \in[0,6]$, and $\eta_{7} \in[-6,6]$, and express them in terms of the $\eta_{i}$. To match the 2 HDMC conventions we define $\gamma \in[-\pi / 2, \pi / 2]$. We refer readers to Refs. $39,51,70,72$ for further details on scanning.

Coupling measurements of the SM-like Higgs can also constrain $c_{\gamma}$ and the couplings $\rho_{i j}$, as seen in Eq.(7). However, $\rho_{t c}$ does not enter any of these observables at leading order, leaving it essentially unconstrained. Allowing for a $2 \sigma$ error bar, we find that even $c_{\gamma} \approx 0.3$ is still allowed by Refs. 73,74 .

There exist indirect constraints on $\rho_{t c}$, which we need to consider before we look at heavy Higgs production at the LHC. For flavor observables, $\rho_{t c}$ enters through loops with charm quarks and a charged Higgs into $B_{s}-\bar{B}_{s}$ mixing and $\mathcal{B}\left(B \rightarrow X_{s} \gamma\right)$. The corresponding limit 75 ,

$$
\left|\rho_{t c}\right| \lesssim 1, \quad \text { for } \quad m_{H^{+}}=300 \mathrm{GeV},
$$

and its counterpart for $m_{H^{+}}=500 \mathrm{GeV}$ is illustrated in Fig. 2, alongside with the EWBGregion. The flavor limit is relatively weak in our general model, in contrast to the type-II 2HDM. For larger $m_{H^{+}}$values, it rapidly becomes irrelevant.

Moreover, finite $c_{\gamma}$ in combination with $\rho_{t c}$ leads to [26] anomalous top decay $t \rightarrow$ 

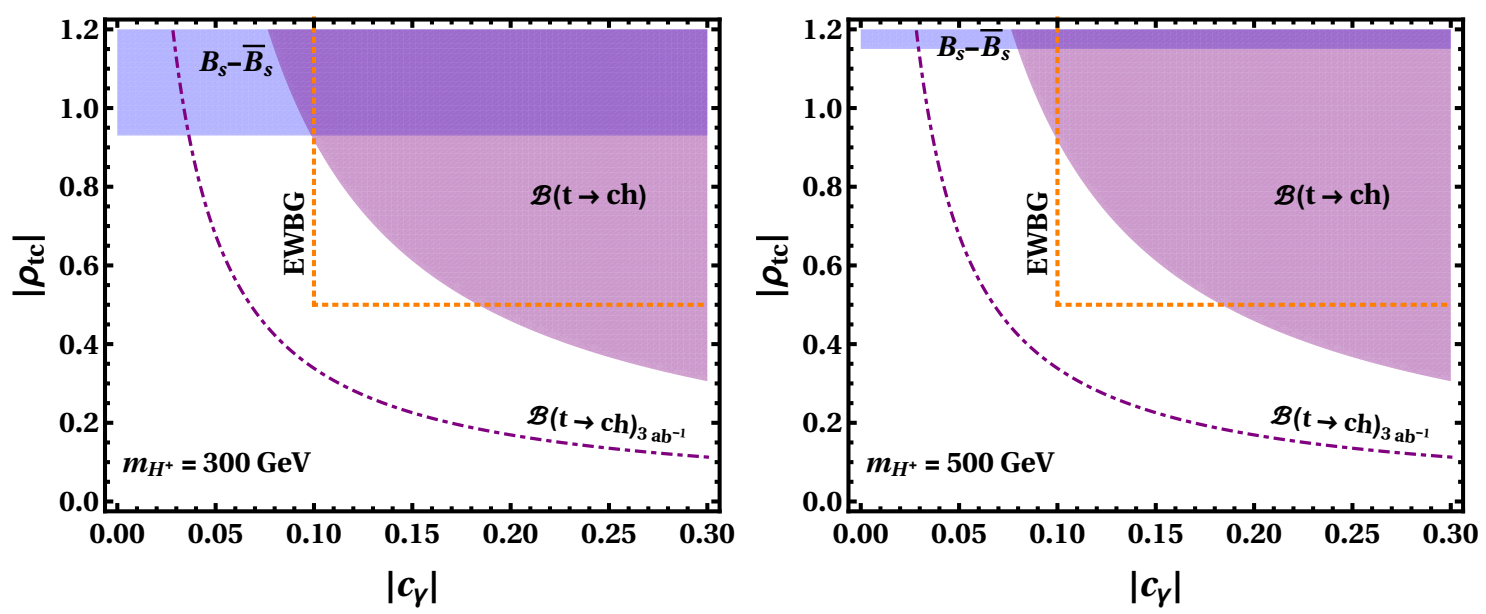

Figure 2: Indirect constraints from $B_{s}-\bar{B}_{s}$ mixing (blue), $\mathcal{B}(t \rightarrow c h$ ) (purple) in the $\rho_{t c}-c_{\gamma}$ plane for two $H^{+}$mass values, together with the baryogenesis region (orange).

ch 28, forbidden at tree level in SM. The current Run 2 limits at 95\%C.L. are

$$
\mathcal{B}(t \rightarrow c h) \approx \frac{c_{\gamma}^{2}\left|\rho_{t c}\right|^{2}}{7.66+c_{\gamma}^{2}\left|\rho_{t c}\right|^{2}}< \begin{cases}1.1 \times 10^{-3} & \text { ATLAS 44] } \\ 4.7 \times 10^{-3} & \text { CMS 440], }\end{cases}
$$

which gets weaker for small $c_{\gamma}$ and vanish in the alignment limit. We illustrate the stronger ATLAS 41] constraint also in Fig. 2, along with the projected HL-LHC 95\%CL upper limit $\mathcal{B}(t \rightarrow c h)<1.0 \times 10^{-4} 76$. If such an anomalous top-charm Yukawa should be observed, the next step in testing electroweak baryogenesis will be to search for new scalar degrees of freedom related to the flavor-changing coupling.

While we will focus on $\rho_{t c}$ throughout this paper, we point out that $\rho_{t u}$ can be tested using a very similar strategy. For the LHC processes discussed in the coming sections, there is always a corresponding process with an up-quark replacing the charm-quark. One difference between the two FCNC scenarios is that $\rho_{t u}$ can induce observable effects in $\mathcal{B}(B \rightarrow \mu \nu)$ [77], within the reach of Belle-II 78. The combination of $\rho_{t c}$ and $\rho_{t u}$ is subject to very strong constraints from $D-\bar{D}$ mixing [75], and we will assume only one of the two, but not both at the same time.

\section{Charged Higgs production}

In the EWBG parameter region of Eq. 99 , the partonic process at LHC

$$
c g \rightarrow b H^{+} \rightarrow b\left(W_{\ell}^{+} h\right) \rightarrow b W_{\ell}^{+} W_{\ell}^{+} W_{\ell}^{-},
$$

probes $\rho_{t c}$ in $H^{+}$-production and $c_{\gamma}$ in $H^{+} \rightarrow W^{+} h$ decay. The production benefits from the relatively large charm density in the proton, as well as the combination [39] with the CKM matrix element $V_{t b}$ following Eq.(7). For a clean analysis, we assume that all three $W$-bosons decay to either electrons or muons. Strictly speaking, the same process can also be induced by $\rho_{c t}$, but this coupling is expected to be much smaller [79] by flavor constraints. The leading-order Feynman diagrams are presented in Fig. 3 . While we will require a tagged $b$-jet, the $b$-inclusive production process could also be defined as $c \bar{b} \rightarrow H^{+} 80,81$. 

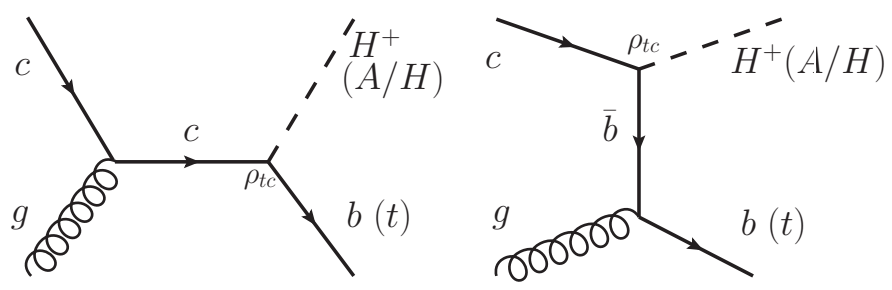

Figure 3: Leading-order Feynman diagrams for the $\rho_{t c}$-induced $c g \rightarrow b H^{+}$and $c g \rightarrow$ $t A / t H$ processes.

The $H^{+} W^{-} h$ coupling, modulated by $c_{\gamma}$, arises from 13,14

$$
\mathcal{L} \supset-\frac{g_{2}}{2} c_{\gamma}\left(h \partial^{\mu} H^{+}-H^{+} \partial^{\mu} h\right) W_{\mu}^{-}+\text {h.c. },
$$

where $g_{2}$ is the $S U(2)$ gauge coupling. To estimate the reach of our charged Higgs signal, we choose two allowed benchmark points,

$$
\rho_{t c}=0.35, \quad c_{\gamma}=0.25, \quad m_{H^{+}}=350,500 \mathrm{GeV},
$$

as given in Tab. 1. For the branching ratios, we ignore the loop-induced decays $H^{+} \rightarrow$ $W^{+} \gamma$ and $H^{+} \rightarrow W^{+} Z$. We generate signal and background events for $\sqrt{s}=14 \mathrm{TeV}$ at leading order with MadGraph5_aMC@NLO [82]. The effective model is implemented in the FeynRules [83 framework, and for parton densities we use NN23LO1 [84]. The events are showered and hadronized with PYTHIA6.4 85] and then handed to Delphes 3.4.2 [86] for a fast detector simulation with the default ATLAS card. Jets are reconstructed with an $R=0.6$ anti- $k_{T}$ algorithm 87 in FastJet [88]. For $b$-tagging as well as $c$-jet and light-jet rejections, we also rely on the default ATLAS card. To allow for extra jets we apply MLM matching [89, 90] with the default MadGraph5_aMC run card. The signal is generated with up to two additional jets.

The dominant SM-backgrounds are $t \bar{t} W$ and $t \bar{t} Z$ production, followed by $W Z+$ jets, $4 t, t \bar{t} h, t Z j, t W Z$, and $Z Z+$ jets. Furthermore, we find the backgrounds $3 t, 3 t+W$, and $3 W$ to be negligible, so we ignore them in our analysis. However, given a mis-identification probability for a jet as a lepton around $10^{-4}$ [91,92], $t \bar{t}$ production will lead to non-trivial background contributions. For all backgrounds, we use the same simulation chain as for the signal, with up to one additional jet for $t \bar{t} W, t \bar{t} Z, W Z+$ jets, $Z Z+$ jets, $t Z+$ jets, $t \bar{t}+$ jets, and no QCD jets for the high-multiplicity backgrounds $4 t, t W Z$ and $t \bar{t} h$. To approximately account for QCD corrections in addition to the jet emission, we attach NLO $K$-factors to the dominant $t \bar{t} V$ backgrounds, namely $1.35\left(W^{-}\right), 1.27\left(W^{+}\right)[93$, and $1.56(Z)$ [94]. We also correct the $W Z+$ jets and $t \bar{t}+$ jets background normalizations to NNLO by factors 2.07 [95] and 1.84 [96] respectively. Furthermore, we adjust the $4 t$, $t \bar{t} h$, and $\bar{t} Z+$ jets rates to NLO through the $K$-factors 2.04 [82, 1.27 [97] and 1.44 [82].

\begin{tabular}{cc|cc|c}
\hline$m_{H^{+}}[\mathrm{GeV}]$ & $\Gamma_{H^{+}}[\mathrm{GeV}]$ & $\mathcal{B}\left(H^{+} \rightarrow c \bar{b}\right)$ & $\mathcal{B}\left(H^{+} \rightarrow W^{+} h\right)$ & $\sigma\left(c g \rightarrow b H^{+}\right)[\mathrm{fb}]$ \\
\hline 350 & 2.2 & 0.85 & 0.15 & 0.126 \\
500 & 3.9 & 0.66 & 0.34 & 0.113 \\
\hline
\end{tabular}

Table 1: Charged Higgs properties for the two benchmark points with $\rho_{t c}=0.35$ and $c_{\gamma}=0.25$. The quoted LHC cross sections include the decay $H^{+} \rightarrow W h$ in the fully leptonic mode, as shown in Eq. 12 , as well as selection and background rejection cuts. 


\begin{tabular}{c|ccccccccc|c}
\hline & $t \bar{t} W$ & $t t \bar{Z}$ & $W Z+$ jets & $4 t$ & $t \bar{t} h$ & $t Z+$ jets & $t W Z$ & $Z Z+$ jets & $t \bar{t}+$ jets & sum bkg \\
\hline merged jets & 1 & 1 & 1 & 0 & 0 & 1 & 0 & 1 & 1 & \\
$K$-factor & NLO & NLO & NNLO & NLO & NLO & NLO & LO & LO & NNLO & \\
$\sigma_{\text {bkg }}[\mathrm{fb}]$ & 0.685 & 0.279 & 0.101 & 0.074 & 0.026 & 0.017 & 0.02 & 0.001 & 0.304 & 1.504 \\
\hline
\end{tabular}

Table 2: Background cross sections for the charged Higgs process after cuts.

The cross sections for the signal and $t W Z$ are kept at LO for simplicity. Here, we simply assume the QCD correction factors for the $W^{+} Z+$ jets and $t Z+$ jets processes to be the same as their respective charge-conjugate processes.

To suppress the backgrounds, we adopt a simple set of requirements. We start with events containing at least three charged leptons and at least one tagged $b$-jet passing

$$
\begin{array}{rlrl}
p_{T, \ell} & >20 \mathrm{GeV}, & \left|\eta_{\ell}\right|<2.5, & \\
p_{T, b} & >20 \mathrm{GeV}, & \left|\eta_{b}\right|<2.5, \\
\Delta R_{i j} & >0.4, \quad(i, j=\ell, b) & & \\
\mathbb{E}_{T} & >35 \mathrm{GeV}, & m_{\ell^{+} \ell^{-}} \not \subset[76,110] \mathrm{GeV}, \quad(\ell=e, \mu) .
\end{array}
$$

The same-flavor opposite-sign dilepton veto reduces the dominant $t \bar{t} Z$ background. In case more than one such $\ell^{+} \ell^{-}$pair exists, we select the combination closest to the $Z$-mass for rejection. The remaining signal rate is given in Tab. 1, while the background rates are summarized in Tab. 2,

For discovery reach and exclusion limits, we compute the significance using the likelihood for a simple counting experiment [98]. If we observe $n$ events with $n_{\text {pred }}$ predicted, the agreement between observation and prediction is given by

$$
Z\left(n \mid n_{\text {pred }}\right)=\sqrt{-2 \ln \frac{L\left(n \mid n_{\text {pred }}\right)}{L(n \mid n)}}, \quad \text { with } \quad L(n \mid \bar{n})=\frac{e^{-\bar{n}} \bar{n}^{n}}{n !} .
$$

For discovery, we compare the observed signal plus background with the background prediction and require $Z(s+b \mid b)>5$. For exclusion, we assume a background-consistent measurement after predicting a signal on top of the background, such that $Z(b \mid s+b)>2$. For instance, assuming an HL-LHC data set with $3000 \mathrm{fb}^{-1}$ and the signal and background cross sections in Tabs. 1 and 2, we find a significance of $\sim 5.6 \sigma$ for $m_{H^{+}}=350 \mathrm{GeV}$ and $\sim 5 \sigma$ for $m_{H^{+}}=500 \mathrm{GeV}$.

We illustrate in Fig. 4 the Run 3 and HL-LHC reach for the charged Higgs signature in the $\left|\rho_{t c}\right|-c_{\gamma}$ plane. We see from the left panel that Run 3 can exclude $\left|\rho_{t c}\right|>0.3$ and $\left|c_{\gamma}\right|=0.27$ for $m_{H^{ \pm}}=350 \mathrm{GeV}$, while the HL-LHC will be sensitive to $\left|\rho_{t c}\right|>0.2$ and $\left|c_{\gamma}\right|=0.14$. For larger Higgs masses, the expected limits become only slightly weaker. The $b$-associated charged Higgs channel covers the $\left|\rho_{t c}\right|$ range preferred by EWBG, but there remains a slice of EWBG parameter space with $\left|c_{\gamma}\right| \lesssim 0.14$. This follows as an effect of decreasing $\mathcal{B}\left(H^{ \pm} \rightarrow W^{ \pm} h\right)$ with smaller $c_{\gamma}$. Unfortunately, this hole is unlikely to be filled by other charged Higgs decays, because for instance the standard signature $H^{+} \rightarrow t \bar{b}$ requires large production rates. 

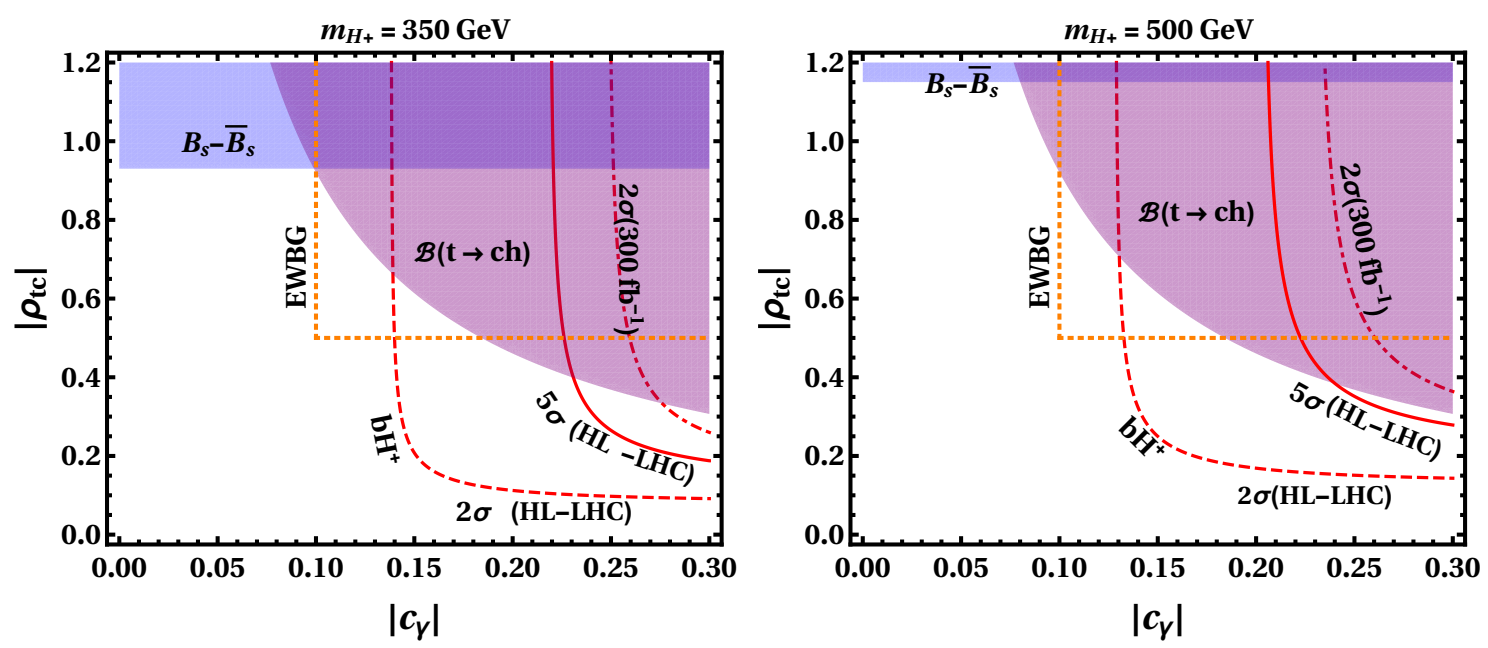

Figure 4: Projected $300 \mathrm{fb}^{-1}$ exclusion (dot-dashed) and HL-LHC discovery (solid) and exclusion (dashed) contours for the charged Higgs signature $p p \rightarrow b H^{+} \rightarrow b W^{+} h$, along with EWBG-favored region and the indirect constraints from Fig. 2.

\section{Neutral Higgs production}

To cover the parameter region $\left|c_{\gamma}\right|<0.14$, left open by the charged Higgs signature, we turn to the neutral Higgs channel,

$$
c g \rightarrow t H / t A \rightarrow t(t \bar{c}),
$$

also given in Fig. 3, where the production and decay are both mediated by $\rho_{t c}$. A very slight $c_{\gamma}$-dependence of the $c g \rightarrow t H / t A \rightarrow t t \bar{c}$ process arises from the heavy Higgs branching ratios. Non-resonant and $t$-channel diagrams with $H / A$ exchange leading to $c c \rightarrow t t$ scattering as well as $g g \rightarrow t t \bar{c} \bar{c}$, though small, are included in our signal analysis.

For small $c_{\gamma}$, the neutral Higgs production process currently leads to the most stringent limit on $\rho_{t c}$ [33. 99], because it affects the SM control region of the Run $2 t t \overline{t t}(4 t)$ analysis by CMS [100]. Based on the number of $b$-jets and leptons, CMS divides its analysis into several signal and two control regions. The most stringent constraint on $\rho_{t c}$ arises from the $t \bar{t} W$ control region (CRW) 32,33. The CMS baseline selection includes two same-sign leptons with

$$
p_{T, \ell}>25,20 \mathrm{GeV} \quad \text { and } \quad\left|\eta_{e}\right|<2.5, \quad\left|\eta_{\mu}\right|<2.4 \text {, }
$$

where the charge-misidentified Drell-Yan background is reduced by vetoing same-sign electron pairs with $m_{e e}<12 \mathrm{GeV}$. The CRW then requires two to five jets, two of them $b$-tagged. All jets have to fulfill $\left|\eta_{j}\right|<2.4$, and events are selected if they fulfill any one of

$$
\begin{array}{rlrl}
\text { (i) } & p_{T, b_{1}}>40 \mathrm{GeV}, \quad p_{T, b_{2}}>40 \mathrm{GeV}, & \\
\text { (ii) } & p_{T, b_{1}}>20 \mathrm{GeV}, \quad p_{T, b_{2}}=20 \ldots 40 \mathrm{GeV}, & p_{T, j_{3}}>40 \mathrm{GeV}, \\
\text { (iii) } & p_{T, b_{1,2}} & =20 \ldots 40 \mathrm{GeV}, & p_{T, j_{3,4}}>40 \mathrm{GeV} .
\end{array}
$$

Finally, the analysis requires 100

$$
H_{T}=\sum_{\text {jets }} p_{T, j}>300 \mathrm{GeV} \quad \text { and } \quad \mathbb{F}_{T}>50 \mathrm{GeV} .
$$




\begin{tabular}{cl|cl|cc}
\hline background & $\sigma[\mathrm{fb}]$ & background & $\sigma[\mathrm{fb}]$ & background & $\sigma[\mathrm{fb}]$ \\
\hline$t \bar{t} W$ & 1.31 & $t \bar{t} Z$ & 1.97 & $t Z+$ jets & 0.007 \\
$4 t$ & 0.092 & $3 t+W$ & 0.001 & $3 t+j$ & 0.0004 \\
$t \bar{t} h$ & 0.058 & charge-flip & 0.024 & non-prompt & $1.5 \times t \bar{t} W$ \\
\hline
\end{tabular}

Table 3: Background cross sections for the dedicated same-sign top search after selection cuts at $\sqrt{s}=14 \mathrm{TeV}$.

With this selection, CMS observes 338 events with $335 \pm 18$ events expected from SMbackgrounds plus $4 t$ signal. To estimate the CRW limits on $\rho_{t c}$, we generate both neutral Higgs processes with the decay $H / A \rightarrow t \bar{c}$, followed by lepton-hadron combinations of the top decays at $\sqrt{s}=13 \mathrm{TeV}$. We use the same setup as for the charged Higgs simulations, except that we use the default CMS detector card in Delphes 3.4.2.

There exist a similar ATLAS search [101], but it is less constraining [102]. This is primarily due to the definition of signal regions and selection criteria. Furthermore, searches for squark pair production in $R$-parity violating supersymmetry [103] and exotics searches for same-sign dileptons and $b$-jets 104 involve similar final states, but their selection cuts are too model-specific to be applied to our signature.

To judge the impact of the existing CMS CRW limits from $4 t$ search, we focus on the border of the EWBG-region with $c_{\gamma}=0.1$ and $\left|\rho_{t c}\right|=0.5$. We stick to our two charged Higgs masses, assume $m_{A} \approx m_{H^{ \pm}}=350,500 \mathrm{GeV}$ for the pseudoscalar, and decouple the heavy scalar $H$. In this scenario, the same-sign top contribution to the CRW arises from $c g \rightarrow t A \rightarrow t t \bar{c}$. We demand that the combination of SM-backgrounds and heavy neutral Higgs production agree with observed within $2 \sigma$ and give the excluded regions in Fig. 5. To scan the parameter space we use a simplified scaling $\left|\rho_{t c}\right|^{2} \mathcal{B}(A \rightarrow t \bar{c})$, such that $\Gamma_{A}=3.05(6.08) \mathrm{GeV}$ for $m_{A}=350(500) \mathrm{GeV}$. The exclusion covers most of the EWBG-region except for small values of $\left|\rho_{t c}\right|$.

A dedicated same-sign top search, such as the $p p \rightarrow t A+X \rightarrow t t \bar{c}+X$ study of Ref. [102], can probe the nominal parameter space of $\rho_{t c}$-EWBG. This process can be searched for in events containing same-sign dileptons $(e e, \mu \mu, e \mu)$, at least three jets with at least two $b$-tag, and some $\mathbb{E}_{T}$. The dominant backgrounds are $t \bar{t} Z, t \bar{t} W$, $4 t$, while $t \bar{t} h$, with $t Z+$ jets, $3 t+W$ and $3 t+j$ give subdominant contributions, and the nonprompt background can be 1.5 times the rate of $t \bar{t} W$. In addition, if a lepton charge gets misidentified, the $t \bar{t}+$ jets and $Z / \gamma^{*}+$ jets processes will also contribute. For further details of the QCD correction factors for different backgrounds, we refer to Ref. [102]. To reduce backgrounds, we applied an event selection different from the CRW of Ref. [100]: the leading and sub-leading same-sign leptons should have $p_{T}>25(20) \mathrm{GeV}$ and $|\eta|<2.5$. All three jets are required to have $p_{T}>20 \mathrm{GeV}$ and $|\eta|<2.5$. All jets and leptons are separated by $\Delta R_{i j}>0.4$. The all event should have $\mathbb{E}_{T}>35 \mathrm{GeV}$. and $H_{T}>$ $300 \mathrm{GeV}$, where the latter includes the two leading sames-sign leptons. The background cross sections after selection cuts are summarized in Tab. 3 .

For the reference values $\left|\rho_{t c}\right|=0.5$ and $c_{\gamma}=0.1$, we generate the same-sign top cross sections for $m_{A}=350$ and $500 \mathrm{GeV}$. Based on the background rates of Tab. 3 and Eq.(16), rescaling the signal cross section by $\left|\rho_{t c}\right|^{2} \mathcal{B}(A \rightarrow t \bar{c})$, we find the exclusion (green dashed) and discovery (green solid) contours in the $\left|c_{\gamma}\right|-\left|\rho_{t c}\right|$ plane as given in Fig. 5 .

A loop hole in the neutral Higgs analysis appears though the destructive interference of $c g \rightarrow t H \rightarrow t t \bar{c}$ and $c g \rightarrow t A \rightarrow t t \bar{c}$. If the widths and masses of the two heavy neutral 

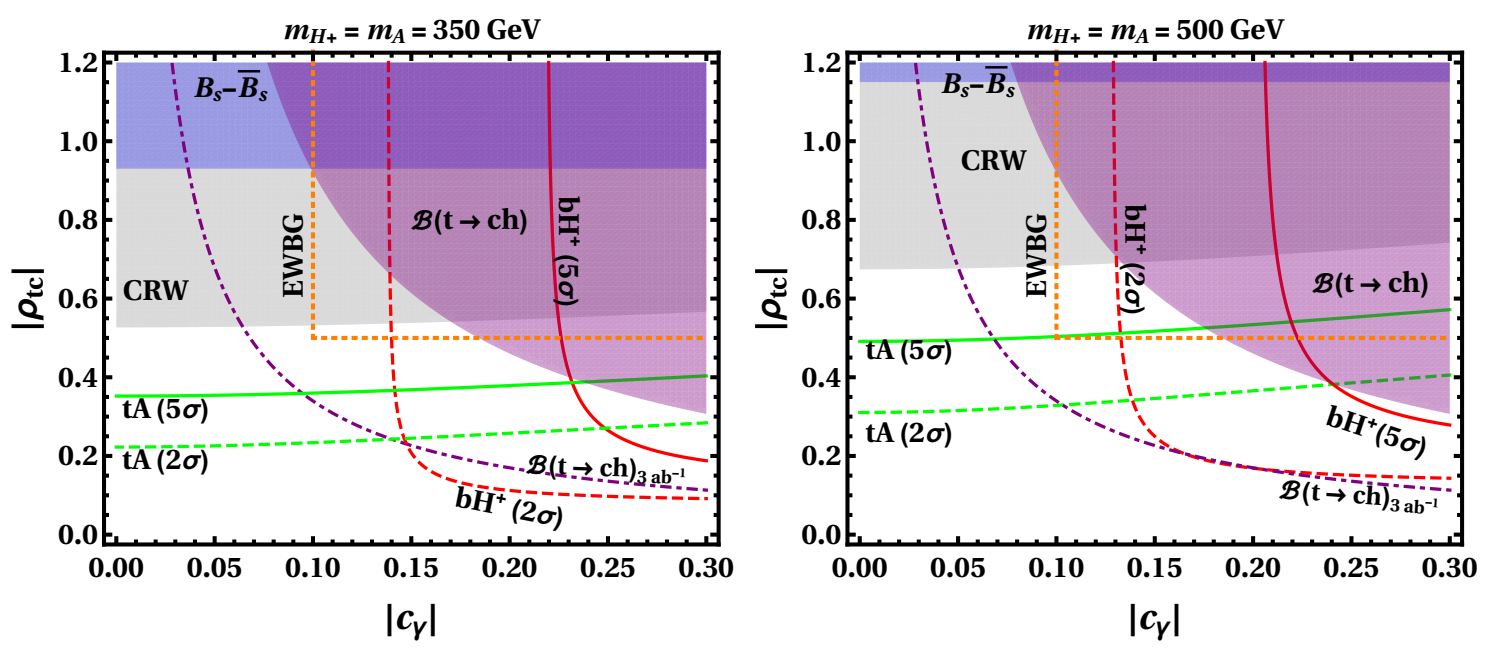

Figure 5: Exclusion regions from neutral Higgs production contributing to the CMS CRW [100] (gray shades), as well as HL-LHC expectations from a dedicated same-sign top search [102] (green). We also show the EWBG region and the indirect constraints from Fig. 2 and the HL-LHC charged Higgs reach from Fig. 4.

Higgses become degenerate, the two production processes completely cancel [32, 33] and the same-sign top signature vanishes. Our limits derived from $A$-production would be similar for $H$-production with $m_{A} \gg m_{H}$. We now illustrate limits for $m_{A} \sim m_{H}$ with a case where the three heavy Higgs masses are of similar size, specifically $m_{H^{ \pm}}=350(500) \mathrm{GeV}$, $m_{A}=343(524) \mathrm{GeV}$, and $m_{H}=355(501) \mathrm{GeV}$. The self-couplings are $\eta_{1}=0.276(0.297)$, $\eta_{2}=1.335(2.762), \eta_{3}=1.66(1.21), \eta_{4}=-0.04(0.398), \eta_{5}=0.121(-0.428), \eta_{6}=$ $-0.181(-0.386), \eta_{7}=0.605(-0.095)$, and $\mu_{22}^{2} / v^{2}=1.189(3.516)$, in agreement with perturbativity, positivity, unitarity, and electroweak precision data [69]. The relevant decays are $A \rightarrow t \bar{c}, Z h$ and $H \rightarrow t \bar{c}, h h, Z Z, W W$, with mild contributions from the $\lambda_{f} c_{\gamma^{-}}$ dependent fermionic decays to $b \bar{b}$ and $t \bar{t}$. For $\rho_{t c}=0.5$ and $c_{\gamma}=0.1$, the total widths are $\Gamma_{A}=3.28(7.37) \mathrm{GeV}$ and $\Gamma_{H}=2.91(6.56) \mathrm{GeV}$, and the combined contributions to the CRW rates are $0.467 \mathrm{fb}$ and $0.261 \mathrm{fb}$, corresponding to 64 and 35.8 events. Demanding that the combination of events expected in the SM and from the neutral Higgs channels agree within $2 \sigma$ of the observed number, we find that $\left|\rho_{t c}\right|=0.5$ is already excluded for $m_{H^{ \pm}}=350 \mathrm{GeV}$ and $c_{\gamma}=0.1$, and barely allowed for $m_{H^{ \pm}}=500 \mathrm{GeV}$. We see that, due to the choice of parameters, the cancellation between $c g \rightarrow t H \rightarrow t t \bar{c}$ and $c g \rightarrow t A \rightarrow t t \bar{c}$ is not exact, and the CRW limit is stronger than the $H$ (or $A$ ) decoupled case.

There exist uncertainties in our results which we have not discussed so far. The $c$-initiated processes $c g \rightarrow b H^{+}$and $c g \rightarrow t A / t H$ have non-negligible systematic uncertainties, such as from parton densities and scale dependence [81, 105 107], which we did not include. Moreover, we do not account for non-prompt and fake backgrounds.

As mentioned in the introduction, we ignore all $\rho_{i j}$ couplings except for $\rho_{t c}$, so before closing we should discuss the impact of this assumption. It may well be that the $\rho^{U, D, L}$ matrices share the flavor-ordering of the Yukawa couplings, $\rho_{t t} \sim \lambda_{t}, \rho_{b b} \sim \lambda_{b}$ and $\rho_{\tau \tau} \sim$ $\lambda_{\tau}$. Current data still allows $\rho_{t t} \lesssim 0.5\left[39\right.$ and $\rho_{b b} \sim 0.1$ [71, 72 for sub-TeV scalars, and both parameters can account for the observed baryon asymmetry. The extra top Yukawa coupling $\rho_{t t}$ can be searched for in signatures such as $g g \rightarrow A / H \rightarrow t \bar{t}$ [108, 109] $g g \rightarrow A / H t \bar{t} \rightarrow t \bar{t} t \bar{t}\left[100\right.$ and $g b \rightarrow \bar{t} H^{+} \rightarrow \bar{t} t \bar{b}$ [80, 81, 110, 111, while rare decays $\mathcal{B}\left(B \rightarrow X_{s} \gamma\right)$ and $B_{d, s}$ mixing provide indirect probes 79. In general, a large value for $\rho_{t t}$ dilutes the decays $A / H \rightarrow t \bar{c}$ and $H^{+} \rightarrow W^{+} h$ through $A / H \rightarrow t \bar{t}$ and $H^{+} \rightarrow t \bar{b}$. However, 
the combination with $\rho_{t c}$ opens additional discovery modes such as $c g \rightarrow t A / t H \rightarrow t t \bar{t}[32$ and $c g \rightarrow b H^{+} \rightarrow b t \bar{b} 39$. There also exist several direct and indirect constraints on $\rho_{b b}$ 71, 72. Finally, a large allowed value of $\rho_{t u}$ 102 combined with non-vanishing $\rho_{t c}$ will be constrained by $D$-meson mixing [75,79]. Similarly, constraints on $\rho_{t t}, \rho_{b b}, \rho_{\tau \tau}$ from flavor physics and low energy observables as discussed in Refs. 38, 72, 75, 79, and their detailed impact on the $\rho_{t c}$-EWBG would be an interesting future direction.

\section{Outlook}

Electroweak baryogenesis is an attractive target for experimental analysis, because it can be tested by a variety of measurements. For any baryogenesis model, it needs to combine new bosonic degrees of freedom with extra $C P$-violation. In our case, the new degrees of freedom are provided by a general or type-III $2 \mathrm{HDM}$. If the Higgs self-couplings are sufficiently large, the heavy Higgs states can be relatively heavy, so we use $m_{H^{+}}=350$ and $500 \mathrm{GeV}$ as benchmark scenarios. The complex phase is given by an FCNC top-charm coupling with $\left|\rho_{t c}\right| \gtrsim 0.5$, combined with a $C P$-even Higgs mixing angle $c_{\gamma} \gtrsim 0.1$. At the LHC, $\rho_{t c}$ has the advantage that we can test it in processes mediated by this large top Yukawa, but with a charm quark in the initial state, while it easily evades the electron EDM constraint.

In the valid $2 \mathrm{HDM}$ parameter space, the charged Higgs has to be relatively light, which means we can search for it via $c g \rightarrow b H^{+}$with a subsequent $H^{+} \rightarrow W^{+} h$ decay. Our proposed analysis is relatively straightforward and probes most of the EWBG parameter space at the HL-LHC, with the exception of small values of $c_{\gamma} \sim 0.1 \ldots 0.12$, when $H^{+} \rightarrow W^{+} h$ decay becomes too suppressed by $C P$-even Higgs boson mixing.

A complementary channel that can survive small $C P$-even Higgs boson mixing is heavy neutral Higgs production, $c g \rightarrow t A / t H$, together with $A / H \rightarrow t \bar{c}$ decay. In this case, production and decay are both mediated by $\rho_{t c}$ without being suppressed by small $c_{\gamma}$, providing strong limits on $\rho_{t c}$ even for small $c_{\gamma}$ values. The search channel at the LHC is same-sign top pairs, allowing us to extract limits already from Run 2. At the HL-LHC, the decay $t \rightarrow c h$, charged heavy Higgs searches, and neutral heavy Higgs searches guarantee a comprehensive coverage of the $\rho_{t c}$-EWBG parameter space in the general $2 \mathrm{HDM}$, leaving us with the challenge of observing the $C P$-violating phase in a dedicated analysis.

\section{Acknowledgments}

First, we would like to thank Kai-Feng (Jack) Chen for discussions and for clarifications on Eq. (19). We are also grateful to Eibun Senaha and Margarete Mühlleitner for very helpful discussions and comments. WSH is supported by MOST 109-2112-M-002-015-MY3 of Taiwan and NTU 109L104019. TM is supported by a Postdoctoral Research Fellowship from Alexander von Humboldt Foundation. The research of TP is supported by the Deutsche Forschungsgemeinschaft (German Research Foundation) under grant 396021762 - TRR 257 Particle Physics Phenomenology after the Higgs Discovery. 


\section{References}

[1] ATLAS, G. Aad et al., Observation of a new particle in the search for the Standard Model Higgs boson with the ATLAS detector at the LHC, Phys. Lett. B 716 (2012) 1, arXiv:1207.7214 [hep-ex].

[2] CMS, S. Chatrchyan et al., Observation of a New Boson at a Mass of $125 \mathrm{GeV}$ with the CMS Experiment at the LHC, Phys. Lett. B 716 (2012) 30, arXiv:1207.7235 [hep-ex]

[3] A. Biekoetter, T. Corbett, and T. Plehn, The Gauge-Higgs Legacy of the LHC Run II, SciPost Phys. 6 (2019) 6, 064, arXiv:1812.07587 [hep-ph],

[4] J. Ellis, C. W. Murphy, V. Sanz, and T. You, Updated Global SMEFT Fit to Higgs, Diboson and Electroweak Data, JHEP 06 (2018) 146, arXiv:1803.03252 [hep-ph].

[5] E. da Silva Almeida, A. Alves, N. Rosa Agostinho, O. J. Éboli, and M. Gonzalez-Garcia, Electroweak Sector Under Scrutiny: A Combined Analysis of LHC and Electroweak Precision Data, Phys. Rev. D 99 (2019) 3, 033001, arXiv:1812.01009 [hep-ph],

[6] S. Kraml, T. Q. Loc, D. T. Nhung, and L. D. Ninh, Constraining new physics from Higgs measurements with Lilith: update to LHC Run 2 results, SciPost Phys. 7 (2019) 4, 052, arXiv:1908.03952 [hep-ph].

[7] S. van Beek, E. R. Nocera, J. Rojo, and E. Slade, Constraining the SMEFT with Bayesian reweighting, SciPost Phys. 7 (2019) 5, 070, arXiv:1906.05296 [hep-ph],

[8] S. Dawson, S. Homiller, and S. D. Lane, Putting standard model EFT fits to work, Phys. Rev. D 102 (2020) 5, 055012, arXiv:2007.01296 [hep-ph],

[9] J. Ellis, M. Madigan, K. Mimasu, V. Sanz, and T. You, Top, Higgs, Diboson and Electroweak Fit to the Standard Model Effective Field Theory, arXiv:2012.02779 [hep-ph],

[10] ATLAS, G. Aad et al., Combined measurements of Higgs boson production and decay using up to $80 \mathrm{fb}^{-1}$ of proton-proton collision data at $\sqrt{\mathrm{s}}=13$ TeV collected with the ATLAS experiment, Phys. Rev. D 101 (2020) 1, 012002, arXiv:1909.02845 [hep-ex].

[11] CMS, A. M. Sirunyan et al., Combined measurements of Higgs boson couplings in proton-proton collisions at $\sqrt{s}=13 \mathrm{TeV}$, Eur. Phys. J. C 79 (2019) 5, 421, arXiv:1809.10733 [hep-ex].

[12] T. Lee, A Theory of Spontaneous T Violation, Phys. Rev. D 8 (1973) 1226.

[13] A. Djouadi, The Anatomy of electro-weak symmetry breaking. I: The Higgs boson in the standard model, Phys. Rept. 457 (2008) 1, arXiv:hep-ph/0503172.

[14] G. Branco, P. Ferreira, L. Lavoura, M. Rebelo, M. Sher, and J. P. Silva, Theory and phenomenology of two-Higgs-doublet models, Phys. Rept. 516 (2012) 1, arXiv:1106.0034 [hep-ph],

[15] A. Djouadi, The Anatomy of electro-weak symmetry breaking. II. The Higgs bosons in the minimal supersymmetric model, Phys. Rept. 459 (2008) 1 ,

arXiv:hep-ph/0503173. 
[16] C. T. Hill and E. H. Simmons, Strong Dynamics and Electroweak Symmetry Breaking, Phys. Rept. 381 (2003) 235, arXiv:hep-ph/0203079. [Erratum: Phys.Rept. 390, 553-554 (2004)].

[17] M. Schmaltz and D. Tucker-Smith, Little Higgs review, Ann. Rev. Nucl. Part. Sci. 55 (2005) 229, arXiv:hep-ph/0502182.

[18] M. Perelstein, Little Higgs models and their phenomenology, Prog. Part. Nucl. Phys. 58 (2007) 247, arXiv:hep-ph/0512128.

[19] J. C. Pati and A. Salam, Lepton Number as the Fourth Color, Phys. Rev. D 10 (1974) 275. [Erratum: Phys.Rev.D 11, 703-703 (1975)].

[20] F. Gursey, P. Ramond, and P. Sikivie, A Universal Gauge Theory Model Based on E6, Phys. Lett. B 60 (1976) 177.

[21] Y. Achiman and B. Stech, Quark Lepton Symmetry and Mass Scales in an E6 Unified Gauge Model, Phys. Lett. B 77 (1978) 389.

[22] R. Barbieri, D. V. Nanopoulos, and A. Masiero, Hierarchical Fermion Masses in E6, Phys. Lett. B 104 (1981) 194.

[23] A. Sakharov, Violation of CP Invariance, C asymmetry, and baryon asymmetry of the universe, JETP Lett. 5 (1967) 24.

[24] A. Bochkarev, S. Kuzmin, and M. Shaposhnikov, Electroweak baryogenesis and the Higgs boson mass problem, Phys. Lett. B 244 (1990) 275.

[25] N. Turok and J. Zadrozny, Electroweak baryogenesis in the two doublet model, Nucl. Phys. B 358 (1991) 471.

[26] K.-F. Chen, W.-S. Hou, C. Kao, and M. Kohda, When the Higgs meets the Top: Search for $t \rightarrow c h^{0}$ at the LHC, Phys. Lett. B725 (2013) 378, arXiv:1304.8037 [hep-ph].

[27] P. Chang, K.-F. Chen, and W.-S. Hou, Flavor Physics and CP Violation, Prog. Part. Nucl. Phys. 97 (2017) 261, arXiv:1708.03793 [hep-ph].

[28] W.-S. Hou, Tree level $t \rightarrow c h^{0}$ or $h^{0} \rightarrow t \bar{c}$ decays, Phys. Lett. B296 (1992) 179.

[29] D. López-Val, T. Plehn, and M. Rauch, Measuring extended Higgs sectors as a consistent free couplings model, JHEP 10 (2013) 134, arXiv:1308.1979 [hep-ph].

[30] J. Haller, A. Hoecker, R. Kogler, K. Mönig, T. Peiffer, and J. Stelzer, Update of the global electroweak fit and constraints on two-Higgs-doublet models, Eur. Phys. J. C 78 (2018) 8, 675, arXiv:1803.01853 [hep-ph],

[31] K. Fuyuto, W.-S. Hou, and E. Senaha, Electroweak baryogenesis driven by extra top Yukawa couplings, Phys. Lett. B 776 (2018) 402, arXiv:1705.05034 [hep-ph].

[32] M. Kohda, T. Modak, and W.-S. Hou, Searching for new scalar bosons via triple-top signature in $c g \rightarrow t S^{0} \rightarrow t t \bar{t}$, Phys. Lett. B 776 (2018) 379, arXiv:1710.07260 [hep-ph],

[33] W.-S. Hou, M. Kohda, and T. Modak, Constraining a Lighter Exotic Scalar via Same-sign Top, Phys. Lett. B 786 (2018) 212, arXiv:1808.00333 [hep-ph]. 
[34] W.-S. Hou, G.-L. Lin, C.-Y. Ma, and C. Yuan, Probing flavor changing neutral Higgs couplings at LHC, Phys. Lett. B 409 (1997) 344, arXiv:hep-ph/9702260.

[35] W. Altmannshofer, J. Eby, S. Gori, M. Lotito, M. Martone, and D. Tuckler, Collider Signatures of Flavorful Higgs Bosons, Phys. Rev. D 94 (2016) 11, 115032 arXiv:1610.02398 [hep-ph],

[36] W. Altmannshofer, B. Maddock, and D. Tuckler, Rare Top Decays as Probes of Flavorful Higgs Bosons, Phys. Rev. D 100 (2019) 1, 015003, arXiv:1904.10956 [hep-ph],

[37] S. Gori, C. Grojean, A. Juste, and A. Paul, Heavy Higgs Searches: Flavour Matters, JHEP 01 (2018) 108, arXiv:1710.03752 [hep-ph].

[38] S. Iguro and K. Tobe, $R\left(D^{(*)}\right)$ in a general two Higgs doublet model, Nucl. Phys. B 925 (2017) 560, arXiv:1708.06176 [hep-ph],

[39] D. K. Ghosh, W.-S. Hou, and T. Modak, Sub-TeV H Boson Production as Probe of Extra Top Yukawa Couplings, Phys. Rev. Lett. 125 (2020) 22, 221801, arXiv:1912.10613 [hep-ph].

[40] CMS, A. M. Sirunyan et al., Search for the flavor-changing neutral current interactions of the top quark and the Higgs boson which decays into a pair of $b$ quarks at $\sqrt{s}=13 \mathrm{TeV}$, JHEP 06 (2018) 102, arXiv:1712.02399 [hep-ex]

[41] ATLAS, M. Aaboud et al., Search for top-quark decays $t \rightarrow H q$ with $36 \mathrm{fb}^{-1}$ of $p p$ collision data at $\sqrt{s}=13$ TeV with the ATLAS detector, JHEP 05 (2019) 123 , arXiv:1812.11568 [hep-ex].

[42] S. Davidson and H. E. Haber, Basis-independent methods for the two-Higgs-doublet model, Phys. Rev. D 72 (2005) 035004, arXiv:hep-ph/0504050, [Erratum: Phys.Rev.D 72, 099902 (2005)].

[43] W.-S. Hou and M. Kikuchi, Approximate Alignment in Two Higgs Doublet Model with Extra Yukawa Couplings, EPL 123 (2018) 1, 11001, arXiv:1706.07694 [hep-ph],

[44] P. Basler and M. Mühlleitner, BSMPT (Beyond the Standard Model Phase Transitions): A tool for the electroweak phase transition in extended Higgs sectors, Comput. Phys. Commun. 237 (2019) 62, arXiv:1803.02846 [hep-ph].

[45] P. Basler, M. Mühlleitner, and J. Müller, Electroweak Phase Transition in Non-Minimal Higgs Sectors, JHEP 05 (2020) 016, arXiv:1912.10477 [hep-ph],

[46] P. Basler, M. Mühlleitner, and J. Müller, BSMPT v2 A Tool for the Electroweak Phase Transition and the Baryon Asymmetry of the Universe in Extended Higgs Sectors, arXiv:2007.01725 [hep-ph].

[47] J. M. Cline, K. Kainulainen, and A. P. Vischer, Dynamics of two Higgs doublet CP violation and baryogenesis at the electroweak phase transition, Phys. Rev. D 54 (1996) 2451, arXiv:hep-ph/9506284.

[48] K. Funakubo, A. Kakuto, S. Otsuki, K. Takenaga, and F. Toyoda, CP violating profile of the electroweak bubble wall, Prog. Theor. Phys. 94 (1995) 845, arXiv:hep-ph/9507452. 
[49] G. Dorsch, S. Huber, T. Konstandin, and J. No, A Second Higgs Doublet in the Early Universe: Baryogenesis and Gravitational Waves, JCAP 05 (2017) 052, arXiv:1611.05874 [hep-ph],

[50] P. Basler, M. Mühlleitner, and J. Wittbrodt, The CP-Violating 2HDM in Light of a Strong First Order Electroweak Phase Transition and Implications for Higgs Pair Production, JHEP 03 (2018) 061, arXiv:1711.04097 [hep-ph],

[51] W.-S. Hou and T. Modak, Prospects for $t Z H$ and $t Z h$ production at the LHC, Phys. Rev. D 101 (2020) 3, 035007, arXiv:1911.06010 [hep-ph],

[52] N. Craig, F. D'Eramo, P. Draper, S. Thomas, and H. Zhang, The Hunt for the Rest of the Higgs Bosons, JHEP 06 (2015) 137, arXiv:1504.04630 [hep-ph].

[53] S. Kanemura, H. Yokoya, and Y.-J. Zheng, Searches for additional Higgs bosons in multi-top-quarks events at the LHC and the International Linear Collider, Nucl. Phys. B 898 (2015) 286, arXiv:1505.01089 [hep-ph].

[54] S. Gori, I.-W. Kim, N. R. Shah, and K. M. Zurek, Closing the Wedge: Search Strategies for Extended Higgs Sectors with Heavy Flavor Final States, Phys. Rev. D 93 (2016) 7, 075038, arXiv:1602.02782 [hep-ph].

[55] S. M. Barr and A. Zee, Electric Dipole Moment of the Electron and of the Neutron, Phys. Rev. Lett. 65 (1990) 21. [Erratum: Phys. Rev. Lett.65,2920(1990)].

[56] K. Fuyuto, W.-S. Hou, and E. Senaha, Cancellation mechanism for the electron electric dipole moment connected with the baryon asymmetry of the Universe, Phys. Rev. D101 (2020) 1, 011901, arXiv:1910.12404 [hep-ph].

[57] ACME, V. Andreev et al., Improved limit on the electric dipole moment of the electron, Nature 562 (2018) 7727, 355.

[58] F. Sala, A bound on the charm chromo-EDM and its implications, JHEP 03 (2014) 061, arXiv:1312.2589 [hep-ph],

[59] K. Funakubo, A. Kakuto, and K. Takenaga, The Effective potential of electroweak theory with two massless Higgs doublets at finite temperature, Prog. Theor. Phys. 91 (1994) 341, arXiv:hep-ph/9310267.

[60] J. M. Cline and P.-A. Lemieux, Electroweak phase transition in two Higgs doublet models, Phys. Rev. D 55 (1997) 3873, arXiv:hep-ph/9609240.

[61] S. Kanemura, Y. Okada, and E. Senaha, Electroweak baryogenesis and quantum corrections to the triple Higgs boson coupling, Phys. Lett. B 606 (2005) 361, arXiv:hep-ph/0411354.

[62] L. Fromme, S. J. Huber, and M. Seniuch, Baryogenesis in the two-Higgs doublet model, JHEP 11 (2006) 038, arXiv:hep-ph/0605242.

[63] D. Borah and J. M. Cline, Inert Doublet Dark Matter with Strong Electroweak Phase Transition, Phys. Rev. D 86 (2012) 055001, arXiv:1204.4722 [hep-ph],

[64] J. M. Cline and K. Kainulainen, Improved Electroweak Phase Transition with Subdominant Inert Doublet Dark Matter, Phys. Rev. D 87 (2013) 7, 071701, arXiv:1302.2614 [hep-ph]. 
[65] G. Dorsch, S. Huber, and J. No, A strong electroweak phase transition in the 2HDM after LHC8, JHEP 10 (2013) 029, arXiv:1305.6610 [hep-ph],

[66] N. Blinov, S. Profumo, and T. Stefaniak, The Electroweak Phase Transition in the Inert Doublet Model, JCAP 07 (2015) 028, arXiv:1504.05949 [hep-ph].

[67] K. Fuyuto and E. Senaha, Sphaleron and critical bubble in the scale invariant two Higgs doublet model, Phys. Lett. B 747 (2015) 152, arXiv:1504.04291 [hep-ph].

[68] P. Basler, M. Krause, M. Muhlleitner, J. Wittbrodt, and A. Wlotzka, Strong First Order Electroweak Phase Transition in the CP-Conserving 2HDM Revisited, JHEP 02 (2017) 121, arXiv:1612.04086 [hep-ph],

[69] D. Eriksson, J. Rathsman, and O. Stal, 2HDMC: Two-Higgs-Doublet Model Calculator Physics and Manual, Comput. Phys. Commun. 181 (2010) 189, arXiv:0902.0851 [hep-ph].

[70] W.-S. Hou, M. Kohda, and T. Modak, Top-assisted di-Higgs boson production motivated by baryogenesis, Phys. Rev. D 99 (2019) 5, 055046, arXiv:1901.00105 [hep-ph],

[71] T. Modak, Probing an additional bottom Yukawa coupling via $b g \rightarrow b A \rightarrow b Z H$ signature, Phys. Rev. D 100 (2019) 3, 035018, arXiv:1905.02137 [hep-ph].

[72] T. Modak and E. Senaha, Probing Electroweak Baryogenesis induced by extra bottom Yukawa coupling via EDMs and collider signatures, JHEP 11 (2020) 025 arXiv:2005.09928 [hep-ph].

[73] CMS Collaboration, Combined Higgs boson production and decay measurements with up to $137 \mathrm{fb}-1$ of proton-proton collision data at sqrts $=13 \mathrm{TeV}$, Tech. Rep. CMS-PAS-HIG-19-005, CERN, Geneva, 2020.

[74] ATLAS Collaboration, A combination of measurements of Higgs boson production and decay using up to $139 \mathrm{fb}^{-1}$ of proton-proton collision data at $\sqrt{\mathrm{s}}=13 \mathrm{TeV}$ collected with the ATLAS experiment, Tech. Rep. ATLAS-CONF-2020-027, CERN, Geneva, Aug, 2020.

[75] A. Crivellin, A. Kokulu, and C. Greub, Flavor-phenomenology of two-Higgs-doublet models with generic Yukawa structure, Phys. Rev. D 87 (2013) 9, 094031, arXiv:1303.5877 [hep-ph],

[76] ATLAS Collaboration, Expected sensitivity of ATLAS to FCNC top quark decays $t \rightarrow Z u$ and $t \rightarrow H q$ at the High Luminosity LHC, Tech. Rep.

ATL-PHYS-PUB-2016-019, CERN, Geneva, Aug, 2016.

[77] W.-S. Hou, M. Kohda, T. Modak, and G.-G. Wong, Enhanced $B \rightarrow \mu \bar{\nu}$ decay at tree level as probe of extra Yukawa couplings, Phys. Lett. B 800 (2020) 135105. arXiv:1903.03016 [hep-ph],

[78] Belle-II, W. Altmannshofer et al., The Belle II Physics Book, PTEP 2019 (2019) 12, 123C01, arXiv:1808.10567 [hep-ex], [Erratum: PTEP 2020, 029201 (2020)].

[79] B. Altunkaynak, W.-S. Hou, C. Kao, M. Kohda, and B. McCoy, Flavor Changing Heavy Higgs Interactions at the LHC, Phys. Lett. B 751 (2015) 135. arXiv:1506.00651 [hep-ph]. 
[80] T. Plehn, Charged Higgs boson production in bottom gluon fusion, Phys. Rev. D 67 (2003) 014018, arXiv:hep-ph/0206121.

[81] E. Boos and T. Plehn, Higgs boson production induced by bottom quarks, Phys. Rev. D 69 (2004) 094005, arXiv:hep-ph/0304034.

[82] J. Alwall, R. Frederix, S. Frixione, V. Hirschi, F. Maltoni, O. Mattelaer, H. S. Shao, T. Stelzer, P. Torrielli, and M. Zaro, The automated computation of tree-level and next-to-leading order differential cross sections, and their matching to parton shower simulations, JHEP 07 (2014) 079, arXiv:1405.0301 [hep-ph].

[83] A. Alloul, N. D. Christensen, C. Degrande, C. Duhr, and B. Fuks, FeynRules 2.0 A complete toolbox for tree-level phenomenology, Comput. Phys. Commun. 185 (2014) 2250, arXiv:1310.1921 [hep-ph].

[84] NNPDF, R. D. Ball, V. Bertone, S. Carrazza, L. Del Debbio, S. Forte, A. Guffanti, N. P. Hartland, and J. Rojo, Parton distributions with QED corrections, Nucl. Phys. B 877 (2013) 290, arXiv:1308.0598 [hep-ph].

[85] T. Sjostrand, S. Mrenna, and P. Z. Skands, PYTHIA 6.4 Physics and Manual, JHEP 05 (2006) 026, arXiv:hep-ph/0603175.

[86] DELPHES 3, J. de Favereau, C. Delaere, P. Demin, A. Giammanco, V. Lemaître, A. Mertens, and M. Selvaggi, DELPHES 3, A modular framework for fast simulation of a generic collider experiment, JHEP 02 (2014) 057, arXiv:1307.6346 [hep-ex].

[87] M. Cacciari, G. P. Salam, and G. Soyez, The anti- $k_{t}$ jet clustering algorithm, JHEP 04 (2008) 063, arXiv:0802.1189 [hep-ph].

[88] M. Cacciari, G. P. Salam, and G. Soyez, FastJet User Manual, Eur. Phys. J. C 72 (2012) 1896, arXiv:1111.6097 [hep-ph].

[89] M. L. Mangano, M. Moretti, F. Piccinini, and M. Treccani, Matching matrix elements and shower evolution for top-quark production in hadronic collisions, JHEP 01 (2007) 013, arXiv:hep-ph/0611129.

[90] J. Alwall et al., Comparative study of various algorithms for the merging of parton showers and matrix elements in hadronic collisions, Eur. Phys. J. C 53 (2008) 473 , arXiv:0706.2569 [hep-ph],

[91] ATLAS, G. Aad et al., Search for supersymmetry at $\sqrt{s}=13$ TeV in final states with jets and two same-sign leptons or three leptons with the ATLAS detector, Eur. Phys. J. C 76 (2016) 5, 259, arXiv:1602.09058 [hep-ex].

[92] E. Alvarez, D. A. Faroughy, J. F. Kamenik, R. Morales, and A. Szynkman, Four Tops for LHC, Nucl. Phys. B 915 (2017) 19, arXiv:1611.05032 [hep-ph].

[93] J. M. Campbell and R. Ellis, $t \bar{t} W^{+-}$production and decay at NLO, JHEP 07 (2012) 052, arXiv:1204.5678 [hep-ph].

[94] J. Campbell, R. K. Ellis, and R. Röntsch, Single top production in association with a Z boson at the LHC, Phys. Rev. D 87 (2013) 114006, arXiv:1302.3856 [hep-ph].

[95] M. Grazzini, S. Kallweit, D. Rathlev, and M. Wiesemann, $W^{ \pm} Z$ production at hadron colliders in NNLO QCD, Phys. Lett. B 761 (2016) 179, arXiv:1604.08576 [hep-ph], 
[96] "twiki: ATLAS-CMS recommended $t \bar{t}$ cross section predictions.",

[97] "twiki: SM Higgs production cross sections at $\sqrt{s}=14$ TeV.".

[98] G. Cowan, K. Cranmer, E. Gross, and O. Vitells, Asymptotic formulae for likelihood-based tests of new physics, Eur. Phys. J. C 71 (2011) 1554, arXiv:1007.1727 [physics.data-an], [Erratum: Eur.Phys.J.C 73, 2501 (2013)].

[99] W.-S. Hou, M. Kohda, and T. Modak, Implications of Four-Top and Top-Pair Studies on Triple-Top Production, Phys. Lett. B 798 (2019) 134953. arXiv:1906.09703 [hep-ph],

[100] CMS, A. M. Sirunyan et al., Search for production of four top quarks in final states with same-sign or multiple leptons in proton-proton collisions at $\sqrt{s}=13 \mathrm{TeV}$, Eur. Phys. J. C 80 (2020) 2, 75, arXiv:1908.06463 [hep-ex],

[101] ATLAS, G. Aad et al., Evidence for tētt production in the multilepton final state in proton-proton collisions at $\sqrt{s}=13$ TeV with the ATLAS detector, Eur. Phys. J. C80 (2020) 11, 1085, arXiv:2007.14858 [hep-ex]

[102] W.-S. Hou, T.-H. Hsu, and T. Modak, Constraining the $t \rightarrow u$ flavor changing neutral Higgs coupling at the LHC, Phys. Rev. D 102 (2020) 5, 055006 , arXiv:2008.02573 [hep-ph],

[103] ATLAS, G. Aad et al., Search for squarks and gluinos in final states with same-sign leptons and jets using $139 \mathrm{fb}^{-1}$ of data collected with the ATLAS detector, JHEP 06 (2020) 046, arXiv:1909.08457 [hep-ex],

[104] ATLAS, M. Aaboud et al., Search for new phenomena in events with same-charge leptons and b-jets in pp collisions at $\sqrt{s}=13$ TeV with the ATLAS detector, JHEP 12 (2018) 039, arXiv:1807.11883 [hep-ex].

[105] M. Buza, Y. Matiounine, J. Smith, and W. van Neerven, Charm electroproduction viewed in the variable flavor number scheme versus fixed order perturbation theory, Eur. Phys. J. C 1 (1998) 301, arXiv:hep-ph/9612398.

[106] F. Maltoni, G. Ridolfi, and M. Ubiali, b-initiated processes at the LHC: a reappraisal, JHEP 07 (2012) 022, arXiv:1203.6393 [hep-ph], [Erratum: JHEP 04, 095 (2013)].

[107] J. Butterworth et al., PDF 4LHC recommendations for LHC Run II, J. Phys. G 43 (2016) 023001, arXiv:1510.03865 [hep-ph].

[108] ATLAS, M. Aaboud et al., Search for Heavy Higgs Bosons A/H Decaying to a Top Quark Pair in pp Collisions at $\sqrt{s}=8 \mathrm{TeV}$ with the ATLAS Detector, Phys. Rev. Lett. 119 (2017) 19, 191803, arXiv:1707.06025 [hep-ex].

[109] CMS, A. M. Sirunyan et al., Search for heavy Higgs bosons decaying to a top quark pair in proton-proton collisions at $\sqrt{s}=13 \mathrm{TeV}$, JHEP 04 (2020) 171, arXiv:1908.01115 [hep-ex].

[110] ATLAS Collaboration, Search for charged Higgs bosons decaying into a top-quark and a bottom-quark at $\sqrt{s}=13$ TeV with the ATLAS detector, Tech. Rep. ATLAS-CONF-2020-039, CERN, Geneva, Aug, 2020. 
[111] CMS, A. M. Sirunyan et al., Search for charged Higgs bosons decaying into a top and a bottom quark in the all-jet final state of pp collisions at $\sqrt{s}=13 \mathrm{TeV}$, JHEP 07 (2020) 126, arXiv:2001.07763 [hep-ex]. 